\title{
Las redes sociales como herramienta de recuperación de la memoria colectiva. Contenido político de la cuenta en Twitter de John F. Kennedy
}

\author{
Sare sozialak: memoria kolektiboa berreskuratzeko \\ tresnak. John F. Kennedyren Twitter \\ kontuaren eduki politikoa
}

\section{Social media as a tool to restore the past. Political content of John F. Kennedy's Twitter account}

\section{Pere Franch ${ }^{1}$ Elena Yeste ${ }^{2}$}

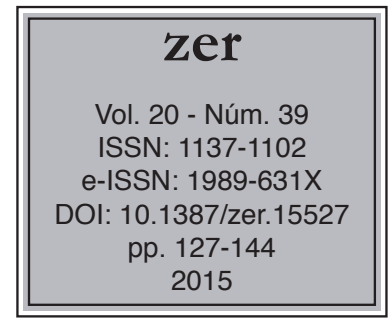

Rebibido el 9 de abril de 2015, aceptado el 3 de noviembre de 2015.

\section{Resumen}

El presente artículo estudia el contenido político de la cuenta de Twitter @JFKsaid, creada por la JFK Library con motivo del cincuenta aniversario de la presidencia de John Fitzgerald Kennedy. Se presenta un análisis de contenido cuantitativo de los temas tratados en los 3.253 tuits publicados cinco décadas después de su mandato. El trabajo analiza cómo se transmite al público actual el legado político de Kennedy, caracterizado por las políticas de bienestar social y defensa de los derechos civiles en el interior y por la guerra fría en el exterior.

Palabras clave: Política, redes sociales, EE.UU., Twitter, John F. Kennedy.

\section{Laburpena}

@JFKsaid Twitter kontua JFK Libraryk sortu zuen John Fitzgerald Kennedyren presidentetzaren 50. urteurrenagatik. Artikulu honetan kontu horren eduki politikoa aztertzen da. Konkretuki, 3.253 txioen eduki-analisi kuantitatiboa egin da. Kennedyren ondare politikoa -gizarte-ongizate politika eta eskubide zibilen defentsa barrualdean, eta gerra hotza kanpoaldean- gaurko publikoari nola helarazten zaion aztertzen da.

Gako-hitzak: Politika, sare sozialak, EE.BB., Twitter, John F. Kennedy.

\footnotetext{
Universitat Ramon Llull, perefp@blanquerna.url.edu

2 Universitat Ramon Llull, elenayp@blanquerna.url.edu
} 


\begin{abstract}
The present article studies the political aspects of the messages published in the Twitter account @JFKsaid, created by the JFK Library to commemorate the fiftieth anniversary of Kennedy's presidency. Through the content analysis methodology, we study quantitatively the political content of the 3.253 messages published in Twitter 50 years after Kennedy's term. We observe how Kennedy's political legacy is transmitted today, whose presidency was characterized by the welfare state and civil rights and involved in Cold War politics.
\end{abstract}

Keywords: Politics, social media, USA, Twitter, John F. Kennedy. 


\section{Introducción}

La Biblioteca-Museo John Fitzgerald Kennedy (JFK Library and Museum), con sede en Boston, en el estado norteamericano de Massachusetts, tiene entre sus objetivos contribuir al estudio y conocimiento de la vida y obra del $35^{\circ}$ presidente de los Estados Unidos, según declara la propia institución en su página web. Para ello, según los responsables de sus colecciones, la JFK Library "acquires, preserves, arranges and describes, and provides access to primary-source materials in all formats that document the life, political career, and legacy of John F. Kennedy" (JFK Library and Museum, 2015).

La JFK Library fue fundada el 5 de diciembre de 1963, a las dos semanas del magnicidio. Casi 50 años después, ya de lleno en el siglo XXI, sus responsables han recurrido a los avances que en el ámbito de la comunicación proporcionan Internet y las redes sociales y han abierto canales en Facebook, Youtube, Itunes, Instagram, Pinterest, Tumblr y Twitter.

En Twitter, que es el objeto de estudio de este artículo, se ofrece acceso a tres cuentas: una es la @JFK Library (https://twitter.com/JFKLibrary), que es la de la institución, activa y vigente en el momento de escribir este artículo; las otras dos son cuentas personales creadas para rememorar la actividad del presidente y de la primera dama.

La cuenta del presidente se abrió el 2 de enero de 2010, cuando se cumplían exactamente 50 años de su anuncio oficial de aspirar a la Casa Blanca. Cuatro días antes, los responsables de comunicación de la JFK Library anunciaban aquella iniciativa diciendo que "culling from campaign schedules, press releases, diary entries and newspapers in the Kennedy Library Archives, the Kennedy1960 Twitter page allows internet users around the world to track the day-to-day campaign schedule of John F. Kennedy's road to the White House" (JFK Library and Museum, 2009). La finalidad era, pues, informar del día a día del presidente y de su reacción ante los hechos de aquella época, con una dinámica propia del siglo XXI: dar a conocer a la opinión pública dónde estaba el presidente, qué hacía o cuál era su posición ante un acontecimiento determinado, como si sus seguidores hubieran podido conocerlo al momento cincuenta años antes. La heredera de aquella primera cuenta es la actual @JFKsaid (https://twitter.com/JFKsaid), que en marzo de 2015 tiene 36.000 seguidores.

La cuenta de Jacqueline Kennedy, @JBK1960 (https://twitter.com/JBK1960), fue creada el 15 de septiembre de 2010 y sólo publicó 98 tuits con extractos de la columna "Campaign wife" que la esposa de Kennedy escribió para diversos periódicos norteamericanos durante la campaña de 1960 (Salsberg, 2010).

El objetivo de este trabajo es conocer el contenido temático de la cuenta de Twitter dedicada a rememorar los 50 años de la presidencia de John Fitzgerald Kennedy. Para ello se han estudiado los tuits publicados en la cuenta @JFKsaid entre el 20 de enero de 2011, cincuenta años después del inicio de su mandato, y el 22 de noviembre de 2013, aniversario de su asesinato. En total, se estudian 3.253 tuits publicados durante dos años, diez meses y dos días, coincidiendo exactamente con el período de su mandato 50 años antes. 
Figura 1. Reproducción del tuit publicado 50 años después de la toma de posesión de John Fitzgerald Kennedy.

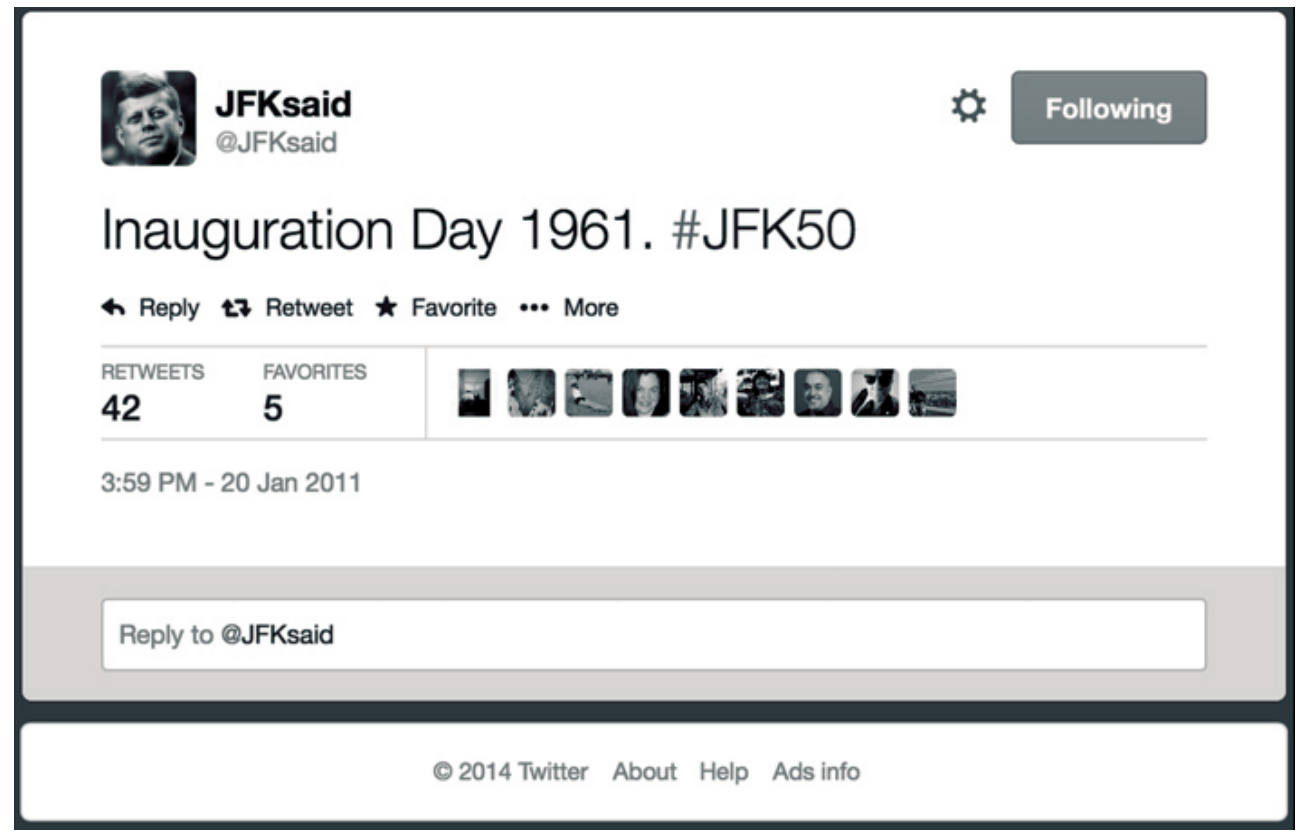

Fuente: JFK Library, cuenta de Twitter @JFKsaid, correspondiente al 20 de enero de 2011.

La finalidad del presente estudio es analizar el contenido de estos 3.253 tuits para conocer sobre qué temas versan y la relación que tiene dicho contenido con lo que aconteció cincuenta años atrás durante los 1.036 días de la presidencia de Kennedy. En concreto, pretendemos observar cómo los contenidos de la cuenta estudiada publicados en el siglo XXI- reflejan lo que ocurrió hace medio siglo. Se trata de ver, en suma, de qué manera los responsables de la JFK Library utilizan Twitter para difundir el legado político del presidente Kennedy, cómo a través de esta red social se explica a las generaciones actuales lo que ocurrió hace 50 años y qué imagen de la vida y obra de Kennedy se pretende transmitir con estos tuits. Para ello se han observado los temas sobre los que versan los mensajes y se ha procedido a una clasificación, para cuantificar el número de tuits dedicados a cada una de las categorías temáticas establecidas. Una vez hecho esto, se ha comparado el número de tuits correspondiente a cada una de las distintas categorías para comprobar a qué temas dedica mayor o menor atención la JFK Library a la hora de difundir el legado de Kennedy a través de Twitter. Y se ha observado la relación que tiene esta distribución temática con los hechos históricos que tuvieron lugar hace 50 años. De hecho, cuando lanzaron esta iniciativa, los responsables de la JFK Library anunciaron que el objetivo era "seguir el recorrido diario de la carrera de John F. Kennedy hacia la Casa Blanca". Comprobar qué relación tiene el relato presentado en Twitter en el siglo XXI con lo que ocurrió a principios de los 60 es el principal fundamento de esta investigación. 


\title{
1. Marco teórico
}

El entorno digital se ha convertido en un nuevo lugar de memoria gracias a un proceso fundamental, la "mediatización de la memoria" (Hoskins, 2009):

In our post-broadcast era, the relationships between media and audiences are transposed and transformed, affording visibility on a new ubiquitous mediatised past, literally a "new memory". (...) Mediatisation has delivered a new self-centred (and immediate) public or semi-public and semi-private documentation and correspondence, in other words a social network memory (Hoskins, 2009: 30).

Los medios digitales han introducido una nueva forma de aproximación a la memoria: "Digital media introduce (...) new means to preserve, restore and represent the past; (...) shape a past that is "always already new, where the old is overwritten by the new" (Hoskins, 2009: 31). Anna Reading (2011: 242) se ha referido a un cambio de paradigma esencial en la conceptualización de la memoria mediada: el "globital memory field: The term 'globital' (...) refers in terms of memory to the synergetic combination of the social and political dynamic of globalization with digitization".

Como explica Paul Longley Arthur (2009: 56-57):

\begin{abstract}
"With Web 2.0 technologies and services, the democratisation of history has taken another huge step forward. Entirely new forms are being developed with the growth of social networking and the possibility of sharing personal information and opinions with a global audience on blogs. Never it has been so easy for people to record their lives for others to see. This is allowing for new kinds of personal expression that are far more public than earlier written records could be, and this is fundamentally altering the old patterns of human communication".
\end{abstract}

En este entorno, "these memories are not simply shared and told but creatively constructed (digital storytelling)" (Garde-Hansen, Hoskins y Reading, 2009: 12). Algunos estudios han explorado el fenómeno del storytelling digital como una nueva forma de representación del 'yo' a través de los nuevos medios digitales: la construcción de los "digitals selves" (Kidd, 2009: 174). Bryan Alexander introduce la especificidad del "Twitter Storytelling": "Twitter's immediacy lends itself to "live" stories" (2011: 61). Como es el caso de la cuenta @JFKsaid, mediante una narración diaria de la actividad del presidente Kennedy entre 1961 y 1963. En este sentido, cabe destacar que "what digital media brings to this representation of the past is a greater personalisation of events, narratives and testimonies." (Garde-Hansen; Hoskins; Reading, 2009: 17).

El uso de las redes sociales por parte de los políticos ha tenido principalmente finalidades electorales. El mejor ejemplo lo encontramos en Barack Obama, cuyo 
éxito en la campaña de 2008 se debió en parte al buen uso que hizo de ellas, ya que le ayudaron a difundir su mensaje político y a ampliar su presencia entre los electores, pero sobre todo a recaudar dinero (Gainous y Wagner, 2011; Clayton, 2010) y a crear una imagen de candidato joven y tecnológicamente experto (Solop, 2010).

El estudio del uso de Twitter en política se ha centrado en la utilización por parte de los usuarios y sobre todo en períodos electorales. La literatura en este ámbito es abundante. Es pionero el trabajo de Tumasjan y sus colegas (2010), que analizaron el uso de Twitter en la campaña electoral alemana de 2009; observaron si los usuarios usaban esta red para el debate político, si los mensajes publicados por ellos reflejaban realmente el sentimiento político del momento y si la presencia de los partidos en Twitter se correspondía con el resultado obtenido en las urnas. A partir de entonces proliferaron los estudios sobre predicciones electorales basadas en Twitter. Congosto y Aragón (2012) señalan los principales. Barberá y Rivero (2012) también estudian el uso de Twitter por parte de los usuarios en las elecciones generales de 2011 en España. En su trabajo intentan determinar en qué grado el uso de esta red social puede constituir una medida de la opinión pública. Small (2011) estudia el contenido político de Twitter y se centra en el uso de los hashtags en Canadá; concluye que la finalidad de Twitter es sobre todo informativa y difusora, y destaca la poca presencia de diálogo entre los usuarios.

En el campo específico del estudio del uso que los dirigentes políticos hacen de Twitter para difundir su mensaje cabe destacar el trabajo de Ammann (2010) sobre el comportamiento en Twitter de los candidatos al Senado de EE.UU. en 2010, en el que observa su actividad cuantitativamente y en relación con el gasto en campaña, sin estudiar el contenido de los tuits. El primer estudio sistemático del contenido de los tuits de los políticos es de Golbeck, Grimes y Rogers (2010). En su trabajo constatan que los congresistas de EE.UU. utilizan esta red de microblogging para difundir información sobre su actividad y especialmente enlaces a sus blogs o a medios que hablan de ellos. De 2010 es el estudio de Glassman, Straus y Shogan sobre el uso de Twitter por parte de los miembros del Congreso estadounidense; entre otros aspectos, estudian el contenido de los tuits publicados, que dividen en ocho categorías temáticas. Los autores reconocen que sus conclusiones ni se pueden generalizar ni a partir de ellas se puede predecir la actitud futura de los congresistas (Glassman et al., 2010).

Sobre el contenido de los tuits de dirigentes políticos concretos, Aharony (2012) estudia cómo los primeros ministros israelí, Benjamin Netanyahu, y británico, David Cameron, y el presidente estadounidense, Barack Obama, usaron Twitter durante tres meses de 2010, centrándose en los mensajes de los líderes hacia la opinión pública pero no en cómo la opinión pública utiliza este medio en su relación con los líderes; en sus conclusiones destaca que los tres utilizaron Twitter para mostrarse más transparentes y llegar a un público más amplio sin la mediación de los medios, pero no para "dialogar" con él, ya que raramente llegan a interactuar con sus seguidores. A la misma conclusión llegan Adams y McCorkindale (2013) en su estudio sobre el uso de Twitter por los candidatos a la presidencia de EE.UU. durante las primarias de 2012. Estas dos autoras han analizado los tuits publicados por los aspirantes a la Casa Blanca entre el 1 y el 29 de febrero de 2012 y afirman que tampoco ellos establecen ningún tipo de diálogo con sus seguidores; sobre los temas tratados, los principales son la economía, hechos de actualidad y las primarias. 
Un trabajo más reciente trata la utilización que Obama hizo de Twitter tras los atentados de Boston de abril de 2013 (Franch, 2013), en el que se constata el escaso uso que hizo el presidente de la red social y su atención principal al control de las armas de fuego, un tema no relacionado con los atentados terroristas.

\section{Metodología}

La metodología utilizada para esta investigación se basa en el análisis de contenido mediante técnicas cuantitativas. Se ha observado el contenido de los 3.253 tuits publicados en la cuenta de Twitter @JFKsaid y se ha llevado a cabo una clasificación temática, esto es, según los temas tratados en el texto publicado. El objetivo es descubrir los temas sobre los que versan los tuits, establecer una clasificación y observar qué cantidad de tuits corresponde a cada tema. La finalidad es obtener datos que se puedan cruzar, contrastar y comparar, para así llegar a conclusiones basadas en la observación empírica del objeto estudiado. Por tanto, el análisis es meramente cuantitativo, técnica que está "destinada a formular, a partir de ciertos datos, inferencias reproducibles y válidas que puedan aplicarse a su contexto" (Krippendorff, 1990: 28) y que Berelson define como "técnica de investigación para la descripción objetiva, sistemática y cuantitativa del contenido manifiesto de la comunicación" (Berelson, 1971, citado por Sánchez Aranda, 2005: 212).

Aun así, si bien el análisis está basado en la comparación cuantitativa de datos, en el proceso de clasificación de los contenidos se recorre a una metodología que tiene cierta analogía con la que en su día fue originada para el análisis cualitativo (sobre todo en los campos de la antropología o la fenomenología social): se trata de la llamada grounded theory o teoría fundamentada en datos (Miles y Huberman 1994; Strauss y Corbin, 1990), que consiste en un proceso heurístico a través del cual se van observando los elementos a estudiar y a medida que van apareciendo datos relevantes se van creando las categorías, que pueden ampliarse o reducirse según van emergiendo nuevos aspectos derivados de la observación. Es por tanto, un método inductivo con el que a partir de la observación empírica de la realidad material (los textos de los tuits en el caso que nos ocupa) se van recopilando elementos para llegar a una conclusión teórica (en este caso acerca del uso de Twitter para difundir la memoria colectiva).

Por tanto, en este estudio los autores hemos ido analizando los tuits para ir creando, a medida que iban apareciendo, las distintas categorías en las que los hemos agrupado. Al final aparecen dos grandes grupos temáticos, según el contenido se refiera a política interior o exterior y en los que se han creado seis y cinco subcategorías respectivamente. Además, aparecen las categorías de tuits dedicados a la carrera espacial, los relativos al asesinato, los que se refieren a conmemoraciones de algún hecho histórico relevante y los que informan de la agenda del presidente (que meramente anuncian un acto, oficial o no, de Kennedy o de alguien de su equipo o familia); mención especial merece la categoría de los tuits agrupados bajo la etiqueta "vida personal o familiar", en los que están aquellos que describen la actividad no específicamente política del presidente o de alguien de su familia, como por ejemplo informar que acude a oír misa, una fotografía en un yate o con su perro, o la constatación de que pasa el fin de semana con su esposa e hijos en su residencia 
de descanso. En cada una de las categorías de tuits dedicados a política interior o exterior aparece también la subcategoría "otros", donde se han clasificado los tuits que no pueden adscribirse a ninguna de las subcategorías restantes y cuya presencia poco significativa impide crear una nueva. En la clasificación general, también aparece la categoría "otros", donde se han incluido los tuits que no pertenecen a ninguna de las anteriores.

Esta es pues, la clasificación temática establecida a través de la observación de los tuits: 1) Política interior, con las seis subcategorías: a) derechos civiles, b) estado del bienestar, c) defensa, d) economía, e) cultura y f) otros; 2) Política exterior, con las cinco subcategorías a) Cuba, b) Vietnam y sudeste asiático, c) Muro de Berlín d) Armas nucleares y relaciones con la URSS y e) otros; 3) Carrera espacial; 4) Conmemoraciones y efemérides; 5) Vida personal y familiar; 6) Agenda; 7) Asesinato y 8) otros.

\section{Resultados y discusión}

El análisis del contenido político de los tuits se ha realizado a partir de la siguiente distribución temática (Tabla 1 y Gráfico 1).

Tabla 1. Temas tratados en la cuenta @JFKsaid.

\begin{tabular}{|c|c|c|c|c|}
\hline TEMA & NÚMERO DE TUITS & $\%$ & & $\%$ \\
\hline Derechos civiles & 223 & 18,13 & & \\
\hline Estado del bienestar & 267 & 21,71 & & \\
\hline Defensa & 137 & 11,14 & & \\
\hline Economía & 192 & 15,61 & & \\
\hline Cultura & 98 & 7,97 & & \\
\hline Otros & 313 & 25,45 & & \\
\hline Política interior & \multicolumn{3}{|c|}{1.230} & 37,81 \\
\hline Cuba & 356 & 29,25 & & \\
\hline Vietnam y sudeste asiático & 62 & 5,09 & & \\
\hline Muro de Berlín & 101 & 8,29 & & \\
\hline $\begin{array}{r}\text { Armas nucleares y relaciones con la } \\
\text { URSS }\end{array}$ & 288 & 23,66 & & \\
\hline Otros & 410 & 33,69 & & \\
\hline Política exterior & \multicolumn{3}{|r|}{1.217} & 37,41 \\
\hline Carrera espacial & \multicolumn{3}{|r|}{119} & 3,66 \\
\hline Conmemoraciones / Efemérides & \multicolumn{3}{|r|}{41} & 1,26 \\
\hline Vida personal y familiar & \multicolumn{3}{|r|}{200} & 6,15 \\
\hline Agenda & \multicolumn{3}{|r|}{330} & 10,14 \\
\hline Asesinato & \multicolumn{3}{|r|}{25} & 0,77 \\
\hline Otros & \multicolumn{3}{|r|}{91} & 2,79 \\
\hline TOTAL & \multicolumn{3}{|r|}{3.253} & \\
\hline
\end{tabular}

Fuente: elaboración propia. 
Gráfico 1. Distribución de los temas de la cuenta @JFKsaid.

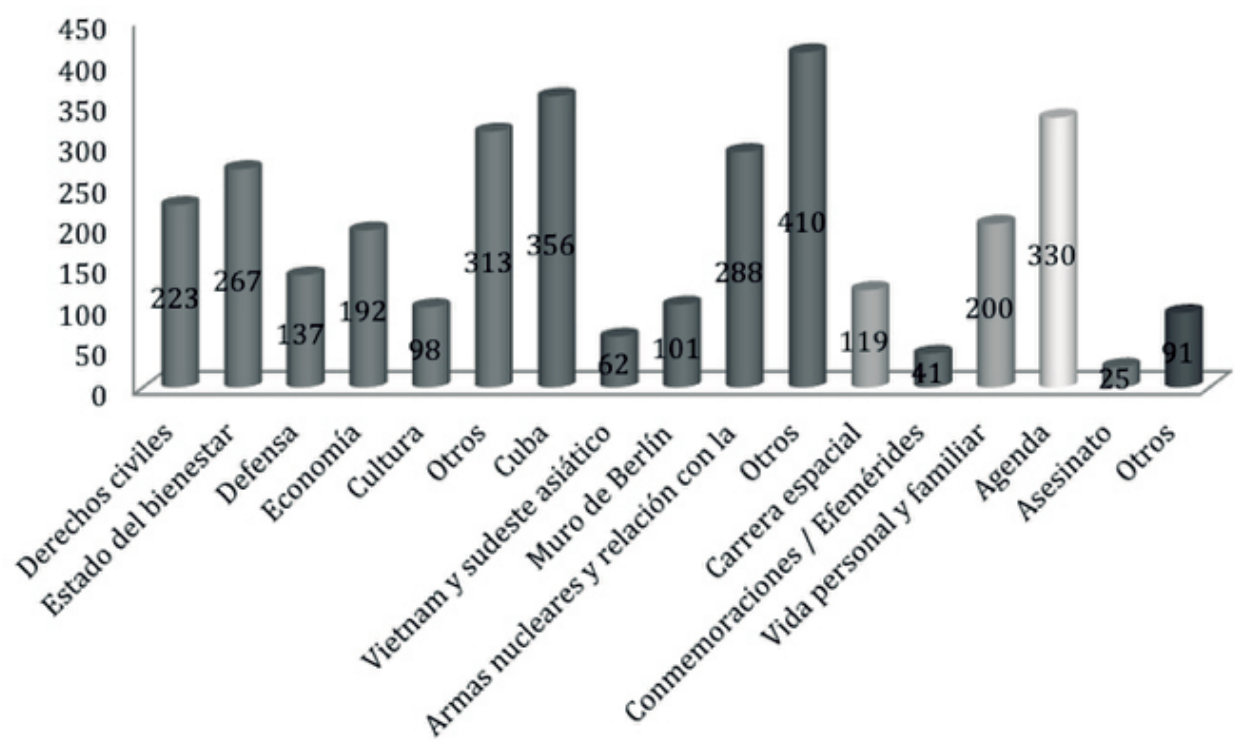

Fuente: elaboración propia.

\subsection{Política interior}

El Estado del bienestar fue un aspecto central en la agenda de la política interior del presidente Kennedy (Altman, 2012), obteniendo el máximo número de tuits de esta categoría de análisis (267 tuits, el 21,71\%) (Gráfico 2). Uno de los aspectos tratados en los tuits sobre política interior es el programa Medicare. "I consider the proposal to provide health insurance for the aged under Social Security one of the most important measures I've advocated", aseguraba Kennedy en una carta dirigida a un senador demócrata referida por la cuenta @JFKsaid (31 de agosto de 2011). Bajo el hashtag \#medicare, la cuenta destacaba, en dos tuits del 20 de mayo de 2012, las siguientes palabras de Kennedy: "I refuse to see this country, and all of us, shrink from these struggles which are our responsibility in our time" y "Because what we are now talking about, in our children's day will seem to be the ordinary business of government". El presidente Lyndon B. Johnson firmó la ley que establecía el programa Medicare en 1965.

Otra de las prioridades de la administración Kennedy en materia de bienestar social fue la Public Welfare Amendments bill, y la legislación para la atención y tratamiento de personas con retraso mental o enfermedades mentales. Así lo refleja la cuenta @JFKsaid con fragmentos de discurso del presidente: "We must strengthen \& improve the programs and facilities serving the mentally ill... the emphasis should be upon timely \& intensive diagnosis"; "I propose a national mental health program to assist in the inauguration of a wholly new emphasis and approach to care for the mentally ill" (5 de febrero de 2013). 
Los derechos civiles de la población norteamericana son el tercer tema con mayor número de tuits $(223$, el 18,13\%) de la cuenta (Yeste y Franch, 2014), después de la categoría Otros ( 313 tuits, el $25,45 \%$ ), en la que se encuentran reunidos todos aquellos tuits que no son clasificables en ninguna de las otras categorías de Política Interior. Mediante el uso de citas literales extraídas de sus declaraciones públicas o comunicados, la cuenta @JFKsaid refleja el discurso político del presidente Kennedy contra la discriminación racial y todas aquellas acciones políticas relacionadas con su agenda diaria y en pro de esta causa, que terminaron con la aprobación de la Ley de Derechos Civiles (Civil Rights Act), el 1 de julio de 1964, y la Ley del Derecho de Voto (Voting Rights Act), el 6 de agosto de 1965. "We are too mixed, this society of ours, to begin to divide ourselves on the basis of race or color", recordaba un tuit del 20 de agosto de 2013. Además, se narran los hechos históricos más relevantes acontecidos durante su mandato, como la marcha sobre Washington, la desegregación de las universidades de Alabama y Mississipi, el asesinato de cuatro niñas negras en un atentado en una iglesia de Birmigham, entre otros.

En materia económica, con 192 tuits (el 15,61\%), la cuenta @JFKsaid presenta a un Kennedy comprometido en la lucha contra la recesión económica iniciada en 1957: "This administration is pledged to safeguard our Nation's economy. It is a vital matter to all of us. Upon it depends our individual wellbeing" (13 de agosto de 2012). Comprometido en la lucha contra el paro: "We cannot be justified until, first, everyone who wants a job can find it..." (18 de noviembre de 2011), y con los jóvenes: "The future promise of any nation can be directly measured by the present prospects of its youth" (14 de febrero de 2013). Comprometido con la lucha contra la discriminación salarial de género: "Signing of the Equal Pay Act, aimed at abolishing wage disparity amongst the genders [Photos] http://ow.ly/lt3vf" (10 de junio de 2013).

La categoría Defensa $(192$, el 15,61\%) recoge la agenda de actos relacionados con el ejército a los que asiste el presidente, que incluyen desde la entrega de medallas de distinción militar, a reuniones en el Pentágono, encuentros con veteranos, con miembros del Ejército, con estudiantes de escuelas militares. También figuran en esta categoría aquellos tuits que contienen fragmentos de discursos de Kennedy en los que se destaca la labor del Ejército: "We are very proud of you and what you represent which is the strong courage of Americans and their determination to defend their country" ( 2 de mayo de 2012).

La categoría Cultura (98 tuits, el 7,97\%) reúne tuits que conciernen la actividad cultural, el arte y los medios de comunicación. Así, esta categoría incluye tuits con referencias a la Educational Television Act de 1962, que impulsó la construcción de emisoras públicas de televisión con fines educativos. Se referencian hechos tales como la llegada de la Mona Lisa a los Estados Unidos en 1963 para su exhibición en el National Gallery of Art. Además de los tuits relativos a la agenda cultural del presidente (inauguraciones, reuniones, asistencia a actos públicos, etc.), están los tuits con comunicados o declaraciones en los que el presidente expresa sus condolencias por autores que han fallecido, como William Faulkner o Robert Frost. 
Gráfico 2. Distribución de temas de Política interior.

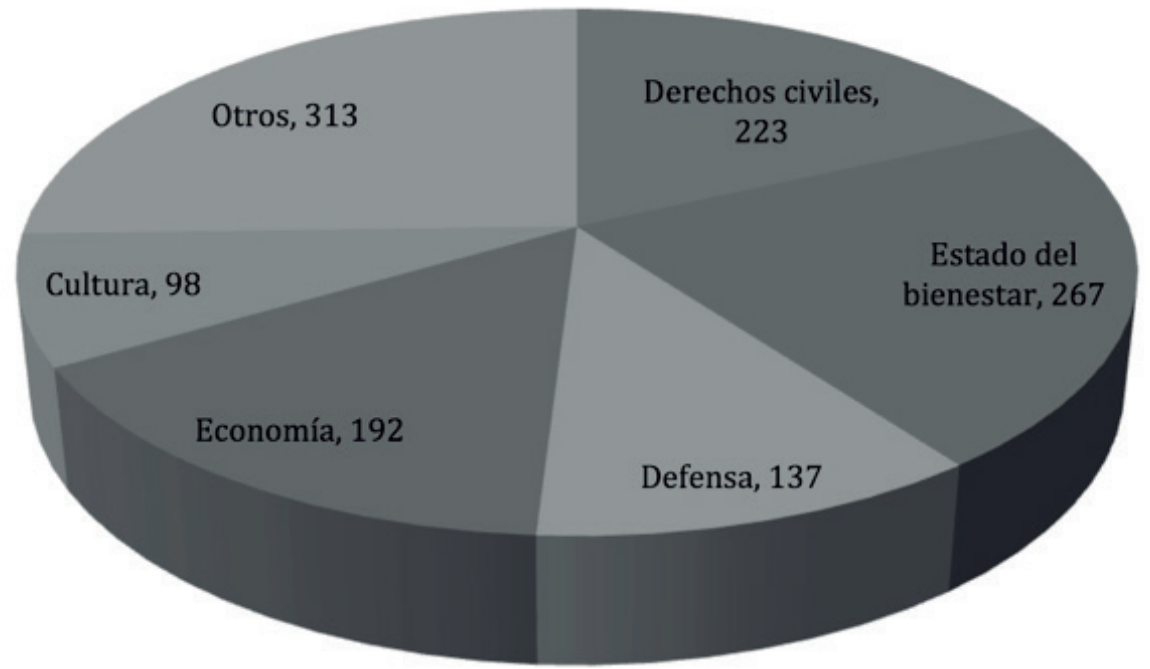

Fuente: elaboración propia.

\subsection{Política exterior}

La cuenta dedica a Cuba casi un tercio $(29,25 \%)$ de los tuits sobre Política exterior (Gráfico 3). Fue bajo el mandato de Kennedy cuando tuvo lugar la crisis de los misiles (octubre de 1962), precedida de la fracasada invasión de la bahía de Cochinos. La cuenta refleja que el presidente asociaba la invasión a la lucha por la libertad: "Where people are denied the right of choice, recourse to such struggle is the only means of achieving their liberties" (18 de abril de 2011) o "I have emphasized before that this is a struggle of Cuban patriots against a Cuban dictator", (20 de abril). Kennedy dejaba clara su posición en el marco de la Guerra Fría cuando tres días después de la invasion declaraba, según un tuit del 20 de abril de 2011: "We do not intend to be lectured on 'intervention' by those whose character was stamped for all time on the bloody streets of Budapest", en referencia a la invasión soviética de Hungría de octubre de 1956.

Las relaciones con Cuba volvieron al primer plano de la actualidad a partir del verano del 1962, con el incremento de la tensión militar que desembocó en la crisis de los misiles, y así lo refleja la cuenta @ JFKsaid. En agosto de 2012 publicó cuatro tuits sobre Cuba. En septiembre, 18, que dejan constancia del aumento de la ayuda militar soviética a Cuba y de la preocupación que ello provocaba en Estados Unidos. Cuando en octubre de 2012 se cumplen 50 años de la llamada crisis de los " 13 días", toda la atención se centra en este tema: aquel mes la cuenta publicó 318 tuits, de los cuales 218 sobre Cuba; esta cifra, 218, significa el 61\% del total de los tuits sobre Cuba publicados. Es más, entre el día 16 de octubre de 2012, cincuenta años después de descubrirse misiles soviéticos en la isla, y el 28 del mismo mes, los 229 tuits publicados son todos sobre Cuba excepto 13, muchos con el hashtag \#13days.

La cuenta permite el acceso a documentos que recogen el contenido de las reuniones del equipo presidencial e incluso ofrece enlaces a grabaciones secretas 
a algunos de los jefes militares: "Shoup, Lemay and Wheeler are left alone to discuss Cuba. They are secretely recorded by a dictaphone. Listen in http://ow.ly/ eBPTp" (19 de octubre de 2012). Otro ejemplo destacable es el tuit que enlaza con el vídeo del canal de Youtube de la JFK que recoge la conversación telefónica de Kennedy con su predecesor Dwight Eisenhower: "Call to President Eisenhower regarding Cuba. Listen in: http://www.youtube.com/watch?v=OjLeFIMC1FQ ...\#13days @eisenhowernews".

La crisis de los misiles de Cuba es inseparable de las relaciones con la URSS y de la carrera nuclear, pero se ha establecido una categoría aparte para pormenorizar el estudio del contenido. En esta categoría hay 288 tuits, el 23,66\% de los dedicados a política exterior. Es relevante que la rememoración de la cumbre de Viena de junio de 1961, la única entre los líderes de las dos superpotencias bajo Kennedy, merezca sólo cuatro tuits, y el único con contenido sustancial se limite a informar que la reunión fue "frank and courteous". Tan poco interés puede ser debido a querer minimizar lo que fue un fracaso rotundo de Kennedy, quien tras el fiasco de Cochinos fue tratado por Jrushchov con cierta superioridad, ya que este le amenazó con cortar el acceso a Berlín (cosa que hizo dos meses después, al construirse el muro) y dijo de él que "el poder no estaba en los Estados Unidos en las firmes manos de un líder” (Fontana, 2011: 261). Otros expertos afirman que en Viena Jrushchov veía a Kennedy como "una persona que se dejaba convencer fácilmente" (Kissinger, 1996: 623).

A la crisis originada con la construcción del muro de Berlín, otro de los grandes temas de la presidencia de Kennedy, la cuenta le dedica 101 tuits, el 8,29\% de los de política exterior. El 12 de agosto de 2011 (ya día 13 en Europa) publicaba: "East German troops close the border between East and West Berlin with barbed wire". A pesar de constituir un hecho determinante en la historia del siglo XX, y con gran valor simbólico, la cuenta no reproduce ninguna declaración de Kennedy hasta el 30 de agosto, cuando se hace eco de su disposición a buscar una solución pacífica a la crisis; de hecho, las declaraciones de Kennedy sobre este tema reproducidas en la cuenta son muy escasas; en cambio, prolifera la reproducción de informaciones de la prensa, siempre del New York Times: 46 de los 101 tuits reproducen textos de este periódico. La cuenta dedica sólo siete tuits a la visita de Kennedy a Berlín del 26 de junio de 1963. Uno publica una foto en el Checkpoint Charlie y otro enlaza con la página de la JFK donde se reproduce su famoso discurso. Los cinco restantes reproducen fragmentos de él, uno la rememorada frase "Two thousand years ago the proudest boast was 'civis Romanus sum'. Today in the world of freedom the proudest boast is 'Ich bin ein Berliner"'.

Sobre Vietnam y el sudeste asiático sólo hay 62 tuits. Esta escasez se explicaría por el hecho de que la escalada militar no se produjo hasta que en agosto de 1964 el presidente Johnson obtuvo del Congreso su apoyo total para la escalada militar: "la resolución del Golfo de Tonkín no era una declaración de guerra, pero dio a Johnson una autoridad casi ilimitada para librarla" (Jones, M. A., 2001: 510). Sin embargo, sí tuvo lugar bajo Kennedy un hecho determinante: el golpe de Estado que acabó con la muerte de Diem y su hermano, el 1 de noviembre de 1963, tras la pérdida de confianza por parte de EE.UU., a lo que contribuyó la represión de las protestas budistas de la primavera de aquel año (Fontana, 2011). 
El resto de mensajes sobre Política exterior aparece en la categoría Otros (410, el $33,69 \%)$, en la que se incluyen una gran variedad de tuits referidos a cualquier aspecto relacionado con algún país extranjero o con las relaciones exteriores de los Estados Unidos (aparte de Cuba, URSS, Berlín o Vietnam), así como los que versan sobre visitas, recepciones y entrevistas con mandatarios extranjeros o viajes de Kennedy.

Gráfico 3. Distribución de temas de Política exterior.

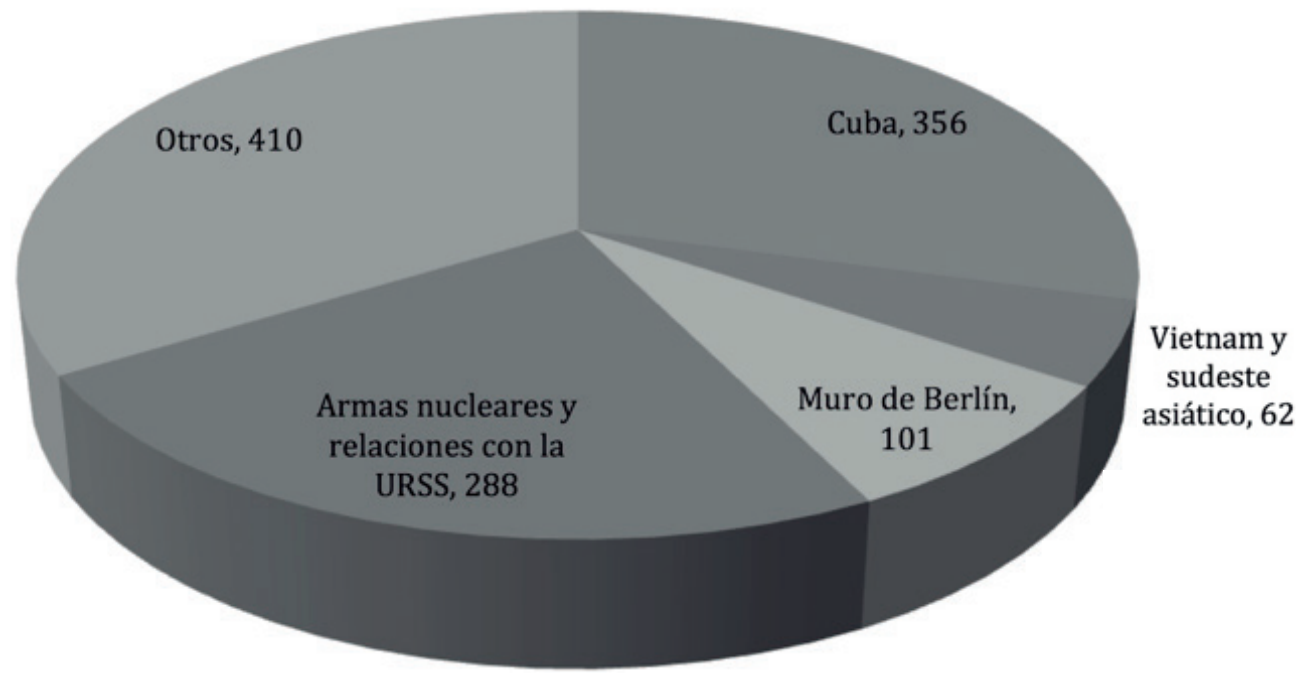

Fuente: elaboración propia.

\subsection{Agenda}

La agenda del presidente Kennedy representa un total de 330 tuits (el 10,14\%). Esta contiene el detalle de todos los actos, reuniones, conferencias de prensa, citas y entrevistas llevados a cabo por el presidente, así como la relación detallada de los desplazamientos en transportes varios realizados. Estos tuits ofrecen detalles tales como el momento de salida y llegada al lugar de destino, o las personas que acompañan al presidente en sus viajes oficiales. La agenda permite seguir la actividad política del presidente, y en muchas ocasiones los tuits contienen enlaces de audio o imagen digitalizados de la JFK Library que ofrecen información adicional sobre el motivo de la actividad o encuentro. Esta categoría incluye la relación de aquellas actividades programadas por el presidente cuyo contenido no está relacionado con ninguna otra de las categorías temáticas definidas en este análisis para la política interior y exterior. Se excluyen de esta categoría todas aquellas actividades personales y familiares, o vinculadas al ocio y tiempo libre del presidente, que, por sus características, se han incluido en otra categoría de análisis.

\subsection{Vida personal y familiar}

Se dedican 200 tuits $(6,15 \%)$ a la vida familiar y personal de los Kennedy. En total hay 53 enlaces a páginas externas, la mayoría a fotos del fondo de la JFK Library. 
Los temas tratados dejan constancia de su carácter, su personalidad o sus costumbres. Por ejemplo, en 23 casos se informa de que el presidente católico asiste a misa. De los 200 tuits de esta categoría, sólo en 14 casos se ofrece información del New York Times, para dar cuenta de detalles tan familiares como que "Kennedy exercising daily to strengthen back" (21 de octubre de 2011) o que su hija Caroline asistía a un jardín de infancia integrado, con niños y niñas negros y blancos (20 de septiembre de 2012).

La mayoría de los tuits dan a conocer aspectos cotidianos de la vida de Kennedy, que serían muy comunes a los de cualquier familia norteamericana: de vacaciones, paseos con su esposa, jugando con sus hijos, con sus perros, en el cine (a ver Spartacus, por ejemplo; 3 de febrero de 2011), a una exposición, practicando deporte (golf, natación), enfermo por un virus, por su espalda o por un resfriado ("Cancels all appointments due to a cold"; 4 de octubre de 2012), o pasando el fin de semana en su residencia. Hay también fotografías de vestidos de Jacqueline, de la limusina oficial, del árbol de Navidad de la Casa Blanca e incluso de los cachorros que parió su perra Pushinka.

\subsection{Carrera espacial}

La cuenta dedica 119 tuits a este tema $(3,66 \%)$. En ellos el mismo Kennedy reconoce que los Estados Unidos iban por detrás de la URSS: "On the space race: 'We started late. (...)" (20 de febrero de 2012). La cuenta refleja que Kennedy justificaba los esfuerzos en la carrera espacial con la defensa de la libertad: "We have vowed that we shall not see [space] governed by a hostile flag of conquest, but by a banner of freedom and peace" (12 de septiembre de 2012).

\subsection{Otros}

La categoría Otros $(91$, el 2,79\%) contiene tuits con informaciones políticas que no están directamente relacionadas con la acción de gobierno del presidente Kennedy, definida en las categorías de política exterior e interior. Una de las principales funciones detectadas en este apartado es de contextualización histórica del periodo analizado en la cuenta. Así, se reúnen informaciones publicadas en el New York Times de hechos acontecidos en otros países, como el siguiente: "NYTIMES: A new economic program announced by the Communist China gives top priority to far-reaching plans for mechanizing farm production" (10 de noviembre de 2012). También se informa de noticias relevantes del momento, como la elección del secretario general de las Naciones Unidas U Thant (1 de diciembre de 2012), o hechos que pueden motivar el interés o la curiosidad de los usuarios, como la llegada al Everest de dos expedicionarios norteamericanos: "NYTIMES: Two members of an American expedition conquered Mount Everest in the first attempt by Americans to scale the 29,028-foot peak" (3 de mayo de 2013).

\subsection{Conmemoraciones / Efemérides}

Esta categoría contiene 41 tuits (el 1,26\%). El 4 de julio de 2012, varios tuits conmemoraban la celebración del Día de la Independencia norteamericana en los que también se apelaba al espíritu de la Constitución de los EE.UU.: "A great new edi- 
fice is not built overnight. It was 11 years from the Declaration of Independence to the writing of the Constitution." Otro significativo momento histórico recordado por la cuenta@JFKsaid es el centenario de Virginia Occidental, que se separó del estado de Virginia durante la Guerra de Secesión y fue admitida por la Unión el 20 de junio de 1863: "The history of W. Virginia, from the day it born in an hour of bitter controversy, has been one of proud determination and steadfast courage" (9 de febrero de 2013). La cuenta @ JFKsaid rememora palabras de Kennedy en relación con algunas eminentes figuras históricas. Es el caso del tuit del 28 de diciembre de 2011 que rememoraba el aniversario del presidente Wilson: "Today marks the birth of Woodrow Wilson, shaper of the first working plan for international cooperation among all peoples of the world".

\subsection{Asesinato}

Sobre el asesinato de Kennedy hay sólo 25 tuits (0,77\%). Son los 25 últimos de la cuenta y 23 de ellos reproducen palabras del periodista de la CBS Walter Cronkite, que informó por televisión del acontecimiento.

\section{Conclusiones}

La función rememorativa de la cuenta@JFKsaid se concreta en varios niveles de análisis de la política llevada a cabo por el presidente John F. Kennedy, así como de las características de su mandato. Asimismo, se realiza una aproximación a la figura de Kennedy, desde un punto de vista personal y familiar, que termina con el asesinato del presidente. De esta manera, la cuenta@JFKsaid recupera, en el siglo XXI, el discurso del presidente Kennedy y la descripción de los hechos más destacados acontecidos durante su mandato.

De acuerdo con el principal objetivo de este estudio, que es conocer el contenido temático de la cuenta de Twitter @JFKsaid, cabe destacar que política exterior y política interior constituyen los dos principales focos de atención temáticos.

Desde el punto de vista de la política interior, la cuenta @ JFKsaid presenta a un Kennedy comprometido con la construcción de un Estado del bienestar y las políticas sociales, con la instauración de un primer sistema sanitario, la defensa de la educación y la lucha contra las desigualdades, sensible a la realidad de las personas mayores, los jóvenes; con el reconocimiento de los derechos civiles de la población negra norteamericana; y con la lucha contra el paro y la situación económica de los EE.UU., en recesión desde 1957.

En cuanto a la política exterior, la cuenta estudiada refleja que la realidad internacional de los años de presidencia de Kennedy estuvieron marcados por la Guerra Fría y que el trato con la URSS y los conflictos derivados de la relación entre los dos bloques fue el principal tema de la agenda exterior del presidente; entre ellos destaca la relación con Cuba, a raíz de la invasión de Cochinos y de la crisis de los misiles, la crisis derivada de la construcción del muro de Berlín y, en muy menor medida, la guerra de Vietnam. El trato marginal que recibe el conflicto del sudeste asiático en la cuenta estudiada corrobora que el conflicto no se convirtió en el principal tema de la agenda exterior de EE.UU. hasta años posteriores. 
La cuenta presenta también un Kennedy con unos hábitos y unas costumbres muy cercanos a los de la familia americana común; con ello, ofrece una imagen popular de la institución presidencial pero a la vez mantiene intacto su prestigio, con lo que se confirma el propósito de consolidar la presidencia como la institución más poderosa del país pero a la vez aproximarla al público, cumpliendo así una de las misiones de los medios sociales. En cuanto a la carrera espacial, a través de la cuenta se constata que el presidente Kennedy era entonces muy consciente del atraso de los EE.UU. respecto a la URSS en este aspecto.

Por otra parte, a partir de lo observado, la cuenta @JFKsaid presenta a un Kennedy que encarna los valores de la seguridad y la defensa nacional, y que cree firmemente en la Constitución y los principios que emanan de ella. En este sentido, en los tuits destaca también la presencia de numerosas referencias que apelan a la historia de la nación norteamericana, tales como la Declaración de Independencia de los EE.UU., la Guerra de Secesión, o el Centenario de la Proclamación de la Emancipación de Lincoln. La función de Twitter, en este caso, es conmemorativa.

Twitter es una herramienta que utilizan los políticos para difundir su mensaje. Lo es, también, para recuperar el mensaje político de dirigentes históricos como John F. Kennedy y difundir su legado y acción política en el presente, como pone de manifiesto la cuenta @JFKsaid. Es decir, como herramienta para la reconstrucción de la memoria colectiva. A ello contribuye la facilidad técnica que las tecnologías digitales ofrecen al usuario para acceder a documentos (textos, fotografía, grabaciones de audio y vídeo) de la época, uno de los cometidos que se propone la JFK Library. De esta manera, un patrimonio que antes era únicamente accesible físicamente, en el archivo tradicional, ahora es compartido por los usuarios en la red, propiciando una nueva forma de socialización de la memoria colectiva, y la oportunidad de interactuar con dicha memoria.

En este trabajo se constata que la dinámica que proporciona Twitter en el siglo XXI y que consiste en dar al usuario la posibilidad de seguir al momento, de manera inmediata, la actividad de un dirigente político, se traslada mediante la cuenta @JFKsaid a la realidad de principios de los 60. Con ello se ofrece al usuario una aproximación a la historia condicionada por la realidad comunicacional actual: una traslación de tiempos y maneras de aprehender el pasado que sin los avances tecnológicos de la era digital sería inimaginable.

\section{Referencias bibiográficas}

ADAMS, Amelia; MCCORKINDALE, Tim (2013). Dialogue and transparency: A content analysis of how the 2012 presidential candidates use Twitter. En: Public Relations Review, vol. 39, $\mathrm{n}^{\circ}$ 4, pp. 357-359.

AHARONY, Noa (2012). Twitter use by three political leaders: an exploratory analysis. En: Online Information Review, vol. 36, $\mathrm{n}^{\circ} 4$, pp. 587-603.

ALEXANDER, Bryan (2011). The New Digital Storytelling: Creating Narratives with New Media. Santa Barbara, California; Denver, Colorado; Oxford, England: Praeger/ABC-CLIO. 
ALTMAN, Nancy J. (2005). The Battle for Social Security: From FDR's Vision To Bush's Gamble. Hoboken, New Jersey: John Wiley \& Sons.

AMMANN, Sky L. (2010). Why Do They Tweet? The Use of Twitter by U.S. Senate Candidates in 2010. En: Social Science Research Network. [http://ssm.com/abstract=1725477]. Fecha de consulta: 9 de abril de 2015.

BARBERÁ, Pablo; RIVERO, Gonzalo (2012). ¿Un tweet, un voto? Desigualdad en la discusión política en Twitter. En: I Congreso Internacional en Comunicación Política y Estrategias de Campaña. Madrid: ALICE [https://files.nyu .edu/pba220/ public/barbera_rivero_2012.pdf]. Fecha de consulta: 9 de abril de 2015.

BERELSON, Bernard (1971). Content Analysis in Communication Research. Nueva York: Hafner Publishing Company. Citado por SÁNCHEZ ARANDA, José J. (2005). Análisis de contenido cuantitativo de medios. En: BERGANZA, Ma Rosa; RUIZ, José A. (coords.) Investigar en comunicación. Madrid: McGrawHill, pp. 207-228.

CLAYTON, Dewey M. (2010). The Presidential Campaign of Barack Obama: A Critical Analysis of a Racially Transcendent Strategy. New York: Routledge.

CONGOSTO, Mª Luz; ARAGÓN, Pablo (2012). Twitter, del sondeo a la sonda: nuevos canales de opinión, nuevos métodos de análisis. En: I Congreso Internacional en Comunicación Política y Estrategias de Campaña. Madrid: ALICE [www.alice-comunicacionpolitica.com/files/ponencias/54-F50000054541342177364-ponencia-1.pdf]. Fecha de consulta: 9 de abril de 2015.

FONTANA, Josep (2011). Por el bien del imperio. Barcelona: Pasado y presente.

FRANCH, Pere (2013). La retórica de Obama ante el terrorismo: estudio del uso de la red social Twitter tras los atentados de Boston de abril de 2013. En: adComunica. Revista Científica de Estrategias, Tendencias e Innovación en Comunicación, $\mathrm{n}^{\mathrm{o}} 6$, pp. 83-101.

GAINOUS, Jason B.; WAGNER, Kevin M. (2011). Rebooting American Politics: The Internet Revolution. Plymouth: Rowman \& Littlefield.

GARDE-HANSEN, Joanne; HOSKINS, Andrew; READING, Anna. (eds.). (2009). Save As... Digital Memories. London: Palgrave Macmillan.

GLASSMAN, Matthew E.; STRAUS, Jacob R.; SHOGAN, Colleen J. (2010). Social Networking and Constituent Communications: Member Use of Twitter During a Two-Month Period in the 111th Congress. En: Congressional Research Service. [http://www.fas.org/sgp/crs/misc/R41066.pdf]. Fecha de consulta: 9 de abril de 2015.

GOLBECK, Jennifer; GRIMES, Justin; ROGERS, Anthony (2010). Twitter use by the US Congress. En: Journal of the American Society for Information Science and Technology, vol. 61, $\mathrm{n}^{\circ}$ 8, pp. 1612-1621.

HOSKINS, Andrew (2009). The Mediatisation of Memory. En: GARDE-HANSEN, Joanne; HOSKINS, Andrew; READING, Anna (eds.). Save As... Digital Memories. London: Palgrave Macmillan, pp. 27-43.

JFK LIBRARY AND MUSEUM (2015). About our collections [http://www.jfklibrary.org/Research/About-Our-Collections.aspx]. Fecha de consulta: 9 de abril de 2015.

JFK LIBRARY AND MUSEUM (2009). News Release: 50 Years Ago - Senator John F. Kennedy of Massachusetts Announces Candidacy for President of the 
United States, 30-12-09. [http://www.jfklibrary.org/About-Us/News-and-Press/ Press-Releases/50-Years-Ago--Senator-John-F-Kennedy-of-Massachusetts-Announces-Candidacy-for-President-of-the-Unite.aspx]. Fecha de consulta: 9 de abril de 2015.

JONES, Maldwyng A. (2001). Historia de Estados Unidos. 1607-1992. Madrid: Cátedra.

KIDD, Jenny (2009). Digital Storytelling and the Performance of Memory. En: GARDE-HANSEN, Joanne; HOSKINS, Andrew; READING, Anna (eds.). Save As... Digital Memories. London: Palgrave Macmillan, pp. 167-183.

KISSINGER, Henry (1996). Diplomacia. Barcelona: Ediciones B.

KRIPPENDORFF, Klaus (1990). Metodología del análisis de contenido. Teoría y práctica. Barcelona: Paidós.

LONGLEY ARTHUR, Paul (2009). Saving Lives: Digital Biography and Life Writing. En: GARDE-HANSEN, Joanne; HOSKINS, Andrew; READING, Anna (eds.) Save As... Digital Memories. London: Palgrave Macmillan, pp. 44-59.

MILES, Matthew B.; HUBERMAN, A. Michael (1994). Quality Data Analysis. An expanded Sourcebook. Thousand Oaks, California: Sage Publications.

SALSBERG, Bob. Jackie Kennedy Joins Twitter 50 Years After JFK Campaign. The Huffington Post, 18-09-10 [http://www.huffingtonpost.com/2010/09/18/jackiejoins-twitter-50-y_n_722063.html]. Fecha de consulta: 9 de abril de 2015.

SMALL, Tamara A. (2011). What the hashtag? A content analysis of Canadian politics on Twitter. En: Information Communication \& Society, vol. 14, $\mathrm{n}^{\circ} 6$, pp. 872-895.

SOLOP, Frederick I. (2010). «RT @BarackObama We just made history»: Twitter and the 2008 Presidential Election. En: HENDRICKS, John A.; DENTON Jr., Robert E. How Barack Obama used new media technology to win the White House. Plymouth: Lexington books.

STRAUSS, Anselm; CORBIN, Juliet M. (1990). Basics of qualitative research: Grounded theory procedures and techniques. Newbury Park, California: Sage Publications.

TUMASJAN, Andranik et al. (2010). Predicting elections with Twitter: what 140 characters reveal about political sentiment. En: Proceedings of the Fourth International Conference on Weblogs and Social Media. Washington D.C.: AAAI Press, pp. 178-185.

YESTE, Elena; FRANCH, Pere (2014). Twitter i comunicació política: estudi del compte de John F. Kennedy. Els drets civils en l'agenda presidencial (19611963). En: Tripodos, $\mathrm{n}^{\circ} 34$, pp. 81-97. 